Article

\title{
Fucoidan Prevents RANKL-Stimulated Osteoclastogenesis and LPS-Induced Inflammatory Bone Loss via Regulation of Akt/GSK3ß/PTEN/NFATc1 Signaling Pathway and Calcineurin Activity
}

\author{
Sheng-Hua Lu ${ }^{1,+}$, Yi-Jan Hsia ${ }^{2,+}$, Kuang-Chung Shih ${ }^{3,4}$ and Tz-Chong Chou ${ }^{5,6,7,8, *}$ \\ 1 Graduate Institute of Life Sciences, National Defense Medical Center, Taipei 114, Taiwan; \\ shooter741109512@gmail.com \\ 2 Dental Department and Devision of Oral and Maxillofacial Surgery, Taipei Tzu Chi Hospital, \\ Buddhist Tzu Chi Medical Foundation, New Taipei City 23142, Taiwan; yjhsia@yahoo.com.tw \\ 3 Division of Endocrinology and Metabolism, Department of Internal Medicine, Tri-Service General Hospital, \\ National Defense Medical Center, Taipei 114, Taiwan; shihkc19610909@gmail.com \\ 4 Division of Endocrinology and Metabolism, Department of Internal Medicine, Cheng-Hsin General Hospital, \\ Taipei 112, Taiwan \\ 5 Department of Biotechnology, Asia University, Taichung 413, Taiwan \\ 6 China Medical University Hospital, China Medical University, Taichung 400, Taiwan \\ 7 Graduate Institute of Medical Sciences, National Defense Medical Center, Taipei 114, Taiwan \\ 8 Department of Pharmacology, National Defense Medical Center, Taipei 114, Taiwan \\ * Correspondence: chou195966@gmail.com; Tel.: +886-2-66289779 (ext. 5357) \\ + These authors have contributed equally to this work.
}

Received: 20 May 2019; Accepted: 5 June 2019; Published: 10 June 2019

check for updates

\begin{abstract}
Excessive osteoclast differentiation and/or function plays a pivotal role in the pathogenesis of bone diseases such as osteoporosis and rheumatoid arthritis. Here, we examined whether fucoidan, a sulfated polysaccharide present in brown algae, attenuates receptor activator of nuclear factor- $\mathrm{kB}$ ligand (RANKL)-stimulated osteoclastogenesis in vitro and lipopolysaccharide (LPS)-induced bone resorption in vivo, and investigated the molecular mechanisms involved. Our results indicated that fucoidan significantly inhibited osteoclast differentiation in RANKL-stimulated macrophages and the bone resorbing activity of osteoclasts. The effects of fucoidan may be mediated by regulation of $\mathrm{Akt} / \mathrm{GSK} 3 \beta / \mathrm{PTEN}$ signaling and suppression of the increase in intracellular $\mathrm{Ca}^{2+}$ level and calcineurin activity, thereby inhibiting the translocation of nuclear factor-activated $\mathrm{T}$ cells $\mathrm{c} 1$ (NFATc1) into the nucleus. However, fucoidan-mediated NFATc1 inactivation was greatly reversed by kenpaullone, a GSK3 $\beta$ inhibitor. In addition, using microcomputer tomography (micro-CT) scanning and bone histomorphometry, we found that fucoidan treatment markedly prevented LPS-induced bone erosion in mice. Collectively, we demonstrated that fucoidan was capable of inhibiting osteoclast differentiation and inflammatory bone loss, which may be modulated by regulation of

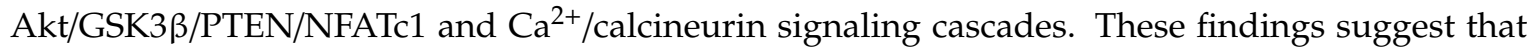
fucoidan may be a potential agent for the treatment of osteoclast-related bone diseases.
\end{abstract}

Keywords: fucoidan; RANKL; osteoclastogenesis; calcineurin; lipopolysaccharide; bone loss

\section{Introduction}

Bone homeostasis is largely controlled by the balance of the actions of bone-resorbing osteoclasts and bone-forming osteoblasts. Activation of osteoclasts and/or dysfunction of osteoblasts results in the development of various bone disorders such as osteoporosis and rheumatoid arthritis [1]. Receptor activator 
of nuclear factor- $\mathrm{KB}$ (RANKL) has been regarded as an important stimulator for osteoclast differentiation from the monocytes/macrophage lineage [2]. After the binding of RANKL to RANK receptor, many transcription factors such as c-fos, NF- $\mathrm{kB}$, and nuclear factor-activated T cells $\mathrm{c1}$ (NFATc1) are activated [3]. Several studies have indicated that NFATc1, in particular, plays an essential role in osteoclast differentiation and functions via upregulation of osteoclast-related genes, including cathepsin $\mathrm{K}$, tartrate-resistant acid phosphatase (TRAP), and matrix metalloproteinase-9 (MMP-9) [4]. As expected, impaired osteoclastogenesis occurs in conditional NFATc1-deficient mice, resulting in osteopetrosis [5].

It is known that RANKL-induced osteoclast differentiation is a complex process that is modulated by multiple pathways [6]. Following stimulation with RANKL, the intracellular level of $\mathrm{Ca}^{2+}\left(\left[\mathrm{Ca}^{2+}\right] \mathrm{i}\right)$ increases, markedly. Subsequently, calcium binds to calmodulin leading to activation of calcineurin, a ubiquitous serine-threonine phosphatase, which dephosphorylates the serine residues in NFATc1 and, in turn, enhances nuclear translocation of NFATc1 [7]. Conversely, RANKL-induced osteoclast differentiation was greatly inhibited by the inhibitors of calcineurin such as FK506 and cyclosporine A [8], suggesting that suppression of the $\mathrm{Ca}^{2+} /$ calmodulin/calcineurin/NFATc1 axis is an effective strategy to attenuate RANKL-induced osteoclastogenesis. Glycogen synthase kinase 3 (GSK3), a serine/threonine kinase, has several biological functions, including regulation of metabolism, transcription, translation, cell growth, and apoptosis [9]. Notably, the GSK3 $\beta$ isoform is capable of phosphorylating NFATc1, resulting in suppression of NFATc1nucleotranslocation and enhancement of nuclear export of NFATc1, ultimately inhibiting osteoclast differentiation [10]. However, Akt-mediated GSK3 $\beta$ phosphorylation inactivates GSK3 $\beta$, thereby promoting nuclear accumulation of NFATc1 and osteoclast differentiation [11,12]. Phosphatase and tensin homolog (PTEN) have been reported to inhibit osteoclastogenesis by suppressing phosphoinositide 3-kinase (PI3K)/Akt signaling via dephosphorylation of PIP3 to PIP2 [13]. In resting cells, GSK3 $\beta$-mediated phosphorylation of PTEN at Thr 366 site activates PTEN, and therefore inhibits the PI3K/Akt pathway. However, under RANKL stimulation, the PI3K/Akt cascade is activated, leading to inactivation of GSK3 $\beta$ and PTEN, leading to osteoclastogenesis [13]. These findings highlight the importance of Akt/GSK3ß/PTEN/NFATc1 and calcium/calcineurin/NFATc1 signaling pathways in the regulation of RANKL-induced osteoclast differentiation.

Currently, bisphosphonates, calcitonin, and estrogen are often used to treat osteoporosis and related fractures [14,15]. However, these pharmacological treatments have serious side effects, including hypercalcemia, increased risk of breast and endometrial cancer, and gastrointestinal intolerance [16], which may limit their use. Therefore, development of safer and more effective drugs or natural products to prevent osteoclast-triggered bone mass loss is urgently needed. Fucoidan, a fucose-containing sulfated polysaccharide present in brown algae, exhibits several beneficial functions, including anti-inflammatory, antioxidant, anticancer, and immunomodulatory activities [17]. Previous studies have reported that fucoidan inhibits RANKL-induced osteoclast formation in bone marrow-derived macrophages (BMMs) mainly through inhibition of NF- $\mathrm{KB}$ activation, a prerequisite for osteoclast differentiation $[18,19]$. However, the exact mechanisms accounting for the inhibition of osteoclastogenesis by fucoidan remain unclear. In this study, we further investigated the molecular mechanisms by which fucoidan affects osteoclast differentiation and inflammatory bone loss, especially focusing on the alterations in Akt/GSK3ß/PTEN/NFATc1 and calcium/calcineurin signaling pathways.

\section{Results}

\subsection{Fucoidan Inhibited RANKL-Induced Osteoclast Differentiation and Bone Resorbing Activity}

RANKL-induced osteoclast differentiation from RAW 264.7 macrophages accompanied by elevation of TRAP-positive multinuclear osteoclasts and their activities were significantly reduced by fucoidan in a dose-dependent manner (Figure 1A). Consistently, RANKL-induced bone resorbing activity of osteoclasts, which was evaluated by pit formation assay, was markedly inhibited by fucoidan (Figure 1B). These findings indicated that fucoidan has an ability to inhibit RANKL-induced osteoclast formation and bone resorbing activity. After cells were treated with different doses of fucoidan (50, 100, or $150 \mu \mathrm{g} / \mathrm{mL}$ ) 
for $48 \mathrm{~h}$, the cell viability evaluated using 3-[4,5-dimethylthiazol-2-yl]-2,5-diphenyl tetrazolium bromide (MTT) analysis was $92.7 \pm 1.2 \%, 93.4 \pm 1.5 \%$, and $93.2 \pm 1.3 \%$, respectively, when compared to that of untreated cells. Thus, the effects of fucoidan at the dose range were not due to its cytotoxicity.

(A)

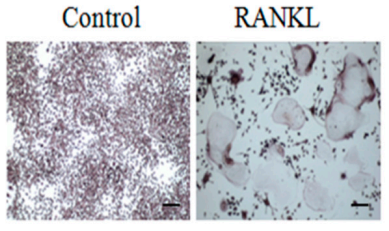

$\mathrm{R}+$ fucoidan $50 \quad \mathrm{R}+$ fucoidan $100 \quad \mathrm{R}+$ fucoidan 150

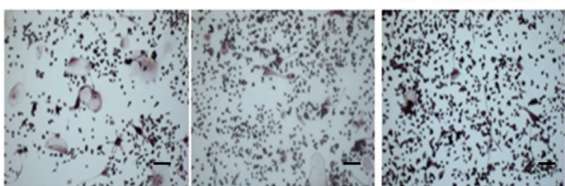

(B)
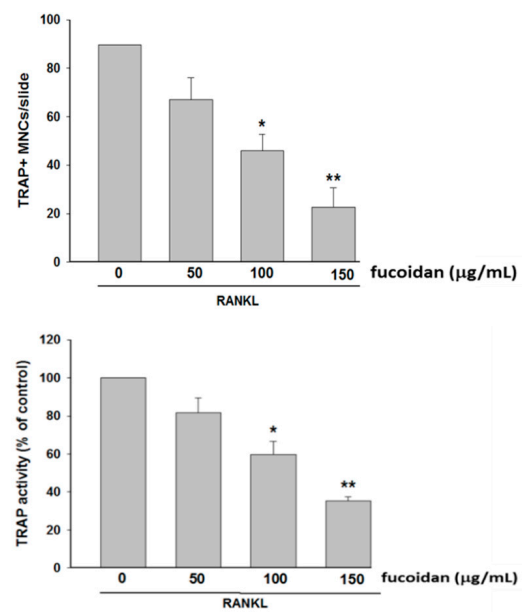

RANKL
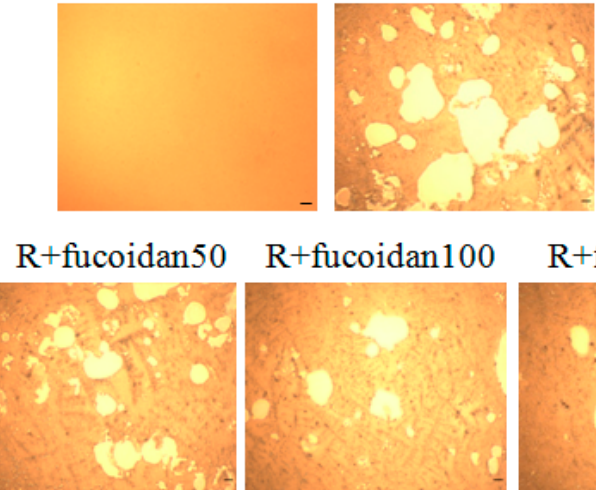

$\mathrm{R}+$ fucoidan 150
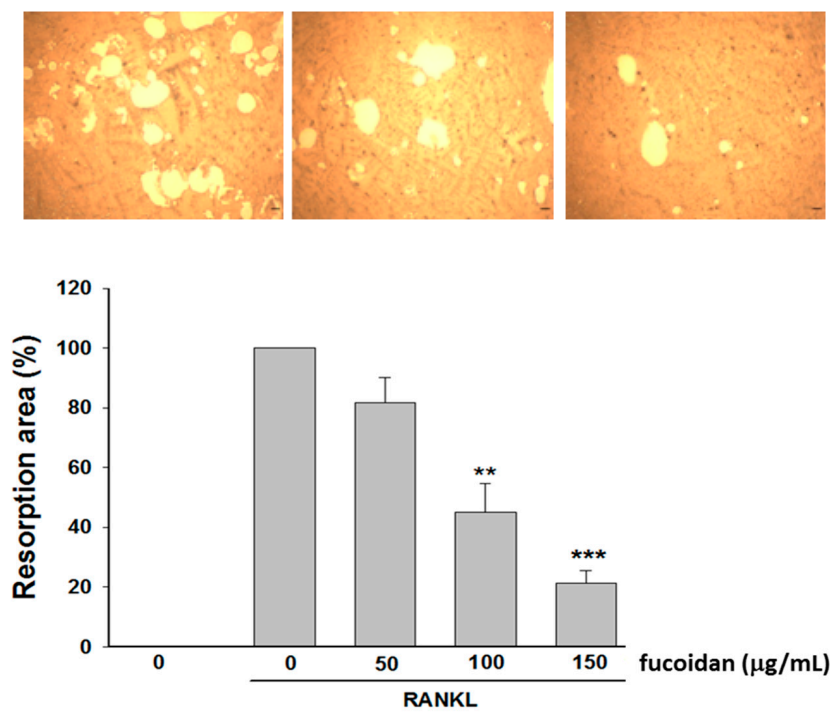

Figure 1. Effects of fucoidan on receptor activator of nuclear factor-kB ligand (RANKL)-induced osteoclast differentiation and bone resorbing activity. RAW 264.7 cells were treated with indicated concentrations of fucoidan in the absence or presence of RANKL $(50 \mathrm{ng} / \mathrm{mL})$ for five days. Then, the cells were fixed and stained with TRAP. The TRAP-positive multinucleated cells (TRAP ${ }^{+}$MNCs) containing three or more nuclei were counted. The TRAP activity was assessed as described in the Method section (A). RAW 264.7 cells were incubated with fucoidan (50, 100, or $150 \mu \mathrm{g} / \mathrm{mL}$ ) for six days in the absence or presence of RANKL ( $50 \mathrm{ng} / \mathrm{mL}$ ). The relative resorption area was expressed as percentage of the values of RANKL-treated alone cells $(B)$. (scale bar $=100 \mu \mathrm{m})$. Data were expressed as mean \pm SD. ${ }^{*} p<0.05,{ }^{* *} p<0.01,{ }^{* * *} p<0.001$ versus RANKL-treated alone cells. 


\subsection{Fucoidan Inhibited RANKL-Activated NFATc1 Nucleotranslocation}

Compared to untreated cells, decreased phospho-NFATc1 (inactive form) in the cytoplasm (Figure 2A) and increased nuclear levels of NFATc1 (Figure 2B) were seen in RANKL-treated cells and the events were greatly inhibited by fucoidan. To examine the role of GSK3 $\beta$, kenpaullone, a GSK3 $\beta$ inhibitor, was added. Our data showed that co-treatment with kenpaullone significantly reversed the actions of fucoidan on NFATc1 phosphorylation and nucleotranslocation. Thus, fucoidan-mediated inhibition of NFATc1 activation is, at least partly, regulated by GSK3 $\beta$-dependent processes.

(A)
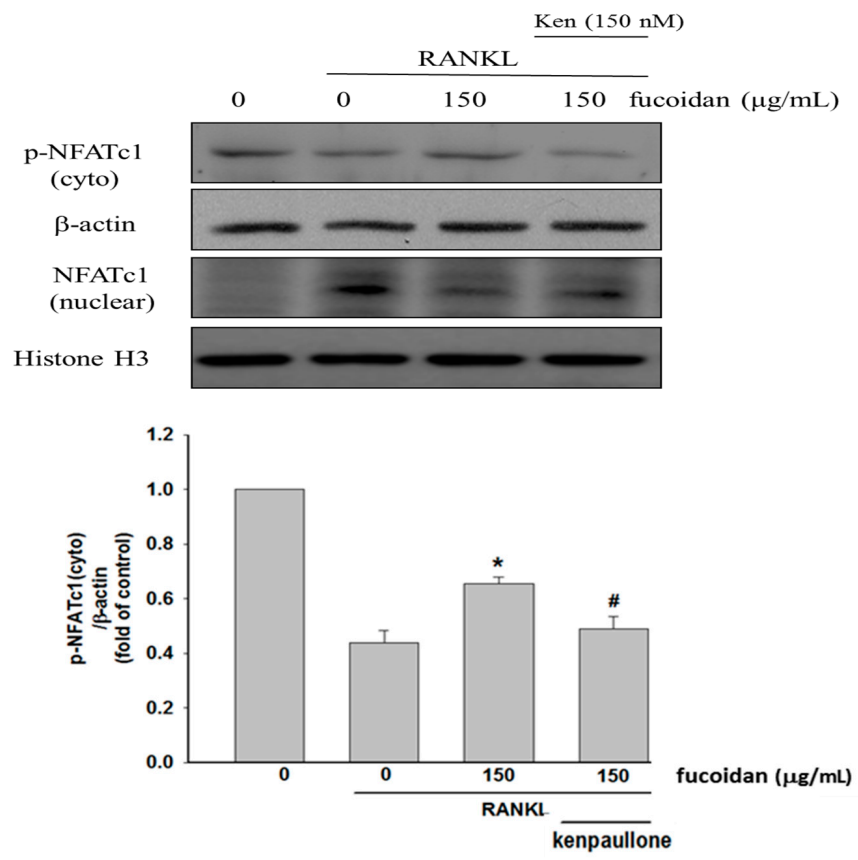

(B)

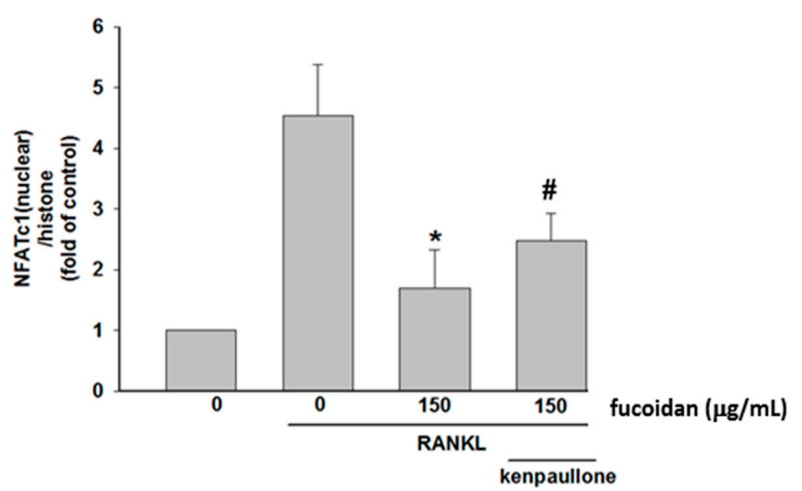

Figure 2. Effects of fucoidan on RANKL-induced NFATc1phosphorylation. Cells were incubated with fucoidan $(150 \mu \mathrm{g} / \mathrm{mL})$ or combination with kenpaullone (Ken, $150 \mathrm{nM})$ followed by addition of RANKL (50 ng/mL) or vehicle for $24 \mathrm{~h}$. The expression of cytoplasmic p-NFATc1 (A) and nuclear NFATc1 expression (B) was determined by Western blotting. Data were expressed as mean \pm SD. ${ }^{*} p<0.05$ versus RANKL-treated alone cells. ${ }^{\#} p<0.05$ versus RANKLand fucoidan group.

\subsection{Fucoidan Regulated the Akt/GSK3//PTEN Cascade}

It is known that activation of Akt results in GSK3 $\beta$ phosphorylation and PTEN inactivation, which ultimately enhances NFATc1 nucleotranslocation and osteoclast differentiation [12,13]. Our data showed that treatment with fucoidan decreased the phosphorylation of GSK3 $\beta$ (Figure 3A) and Akt but increased PTEN phosphorylation (Figure 3B) compared to that of RANKL-treated alone cells. 
Accordingly, fucoidan-mediated GSK3 $\beta$ activation may result from suppressing Akt phosphorylation due to increased PTEN phosphorylation by GSK3 $\beta$.

(A)
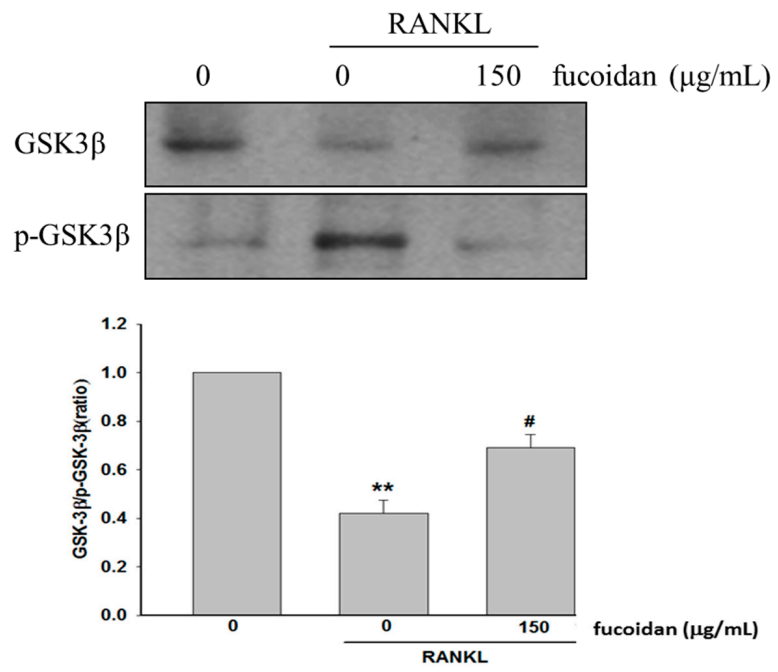

(B)
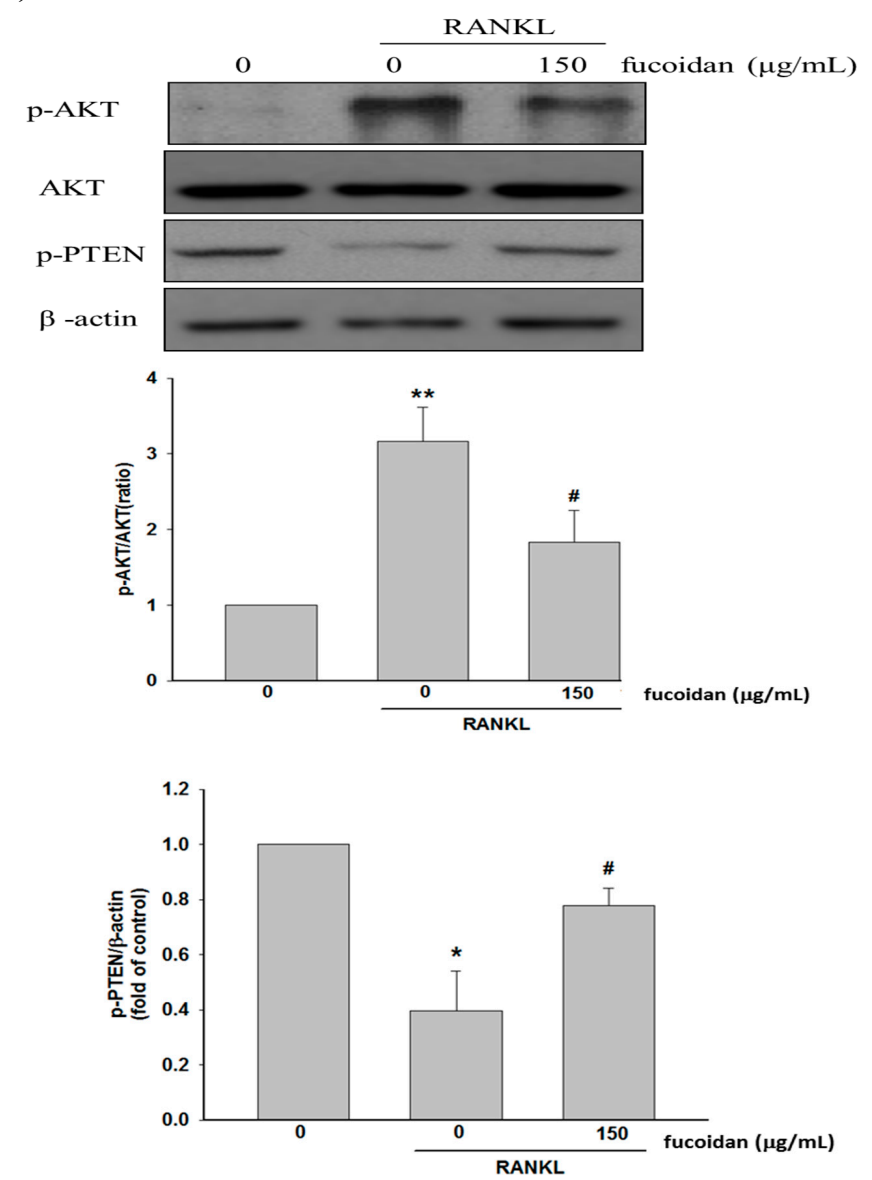

Figure 3. Effects of fucoidan on the expression of GSK3 $\beta$, p-GSK3 $\beta$, p-Akt, Akt, and p-PTEN expression. RAW 264.7 cells were pretreated with fucoidan $(150 \mu \mathrm{g} / \mathrm{mL})$ for $2 \mathrm{~h}$ followed by addition of RANKL $(50 \mathrm{ng} / \mathrm{mL}$ ) or vehicle for $24 \mathrm{~h}$ and the protein expression of GSK3 $\beta$ and p-GSK3 $\beta$ was determined (A). After treatment with RANKL or vehicle for $15 \mathrm{~min}$, the expression of p-AKT, Akt, and p-PTEN of various groups was determined (B). Data were expressed as mean $\pm \mathrm{SD}$. ${ }^{*} p<0.05,{ }^{* *} p<0.01$ versus untreated cells. ${ }^{\#} p<0.05$ versus RANKL-treated alone cells. 


\subsection{Fucoidan Inhibited RANKL-Evoked $\left[\mathrm{Ca}^{2+}\right]$ i and Calcineurin Activity}

RANKL-stimulated increase in $\left[\mathrm{Ca}^{2+}\right] \mathrm{i}$ and calcineurin activity has been considered to enhance NFATc1 nuclear translocation via dephosphorylation of NFATc1 [8]. RANKL-induced increase in $\left[\mathrm{Ca}^{2+}\right]$ i (Figure $4 \mathrm{~A}$ ) and calcineurin activity (Figure $4 \mathrm{~B}$ ) were greatly inhibited by fucoidan. These results suggest that suppressing calcium and calcineruin cascade was an important mechanism contributing to the reduction of NFATc1 activation.

(A)
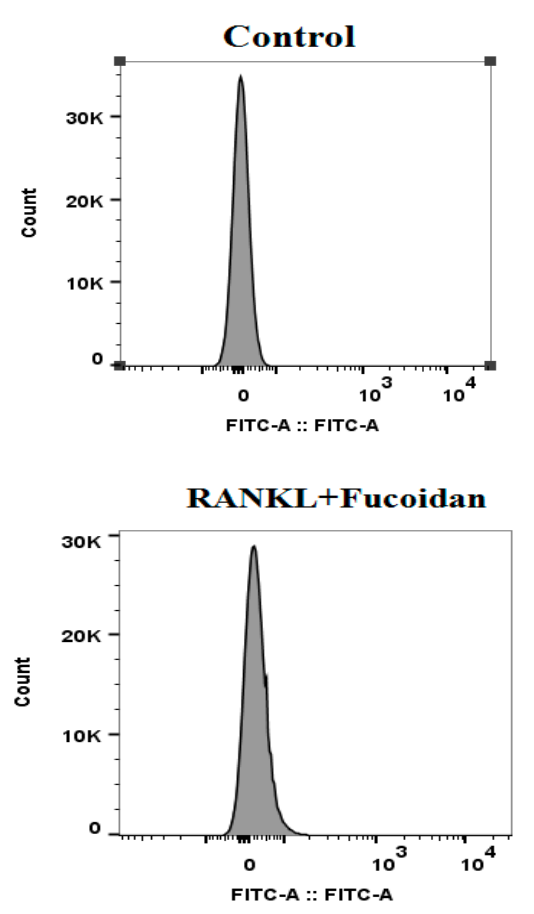

(B)
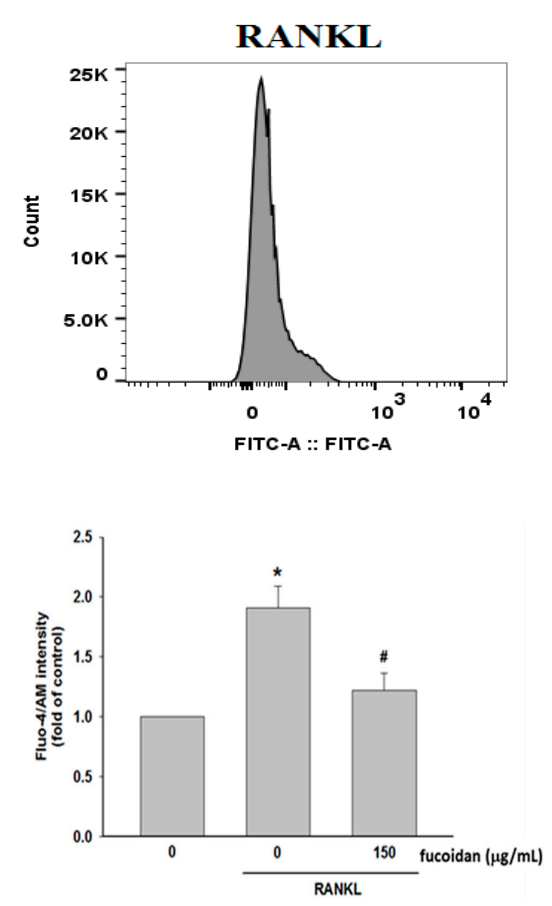

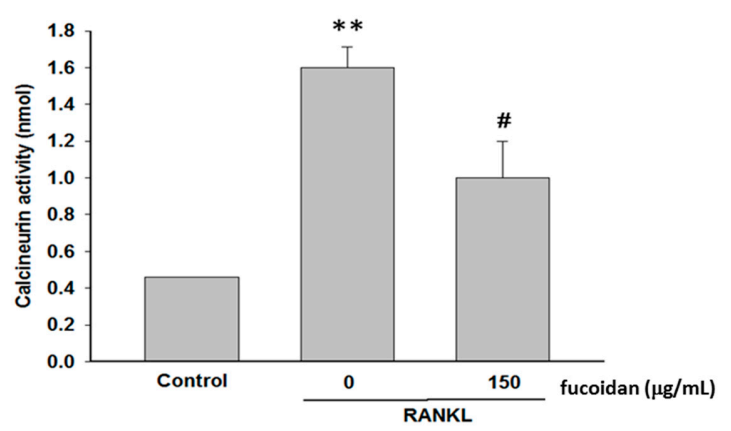

Figure 4. Effects of fucoidan on $\left[\mathrm{Ca}^{2+}\right] \mathrm{i}$ and calcineurin activity in RANKL-stimulated RAW 264.7 cells. Cells pretreated with fucoidan $(150 \mu \mathrm{g} / \mathrm{mL})$ for $2 \mathrm{~h}$ were incubated with Fluo-4/AM $(5 \mu \mathrm{M})$ for $30 \mathrm{~min}$ followed by stimulation with RANKL $(50 \mathrm{ng} / \mathrm{mL}$ ) for $10 \mathrm{~min}$ before the analysis by flow cytometry (A). Cells were treated with RANKL for $30 \mathrm{~min}$ in the absence or presence of fucoidan, and the cell homogenates were prepared for calcineurin activities assay (B). Data were expressed as mean \pm SD. ${ }^{*} p<0.05,{ }^{* *} p<0.01$ versus untreated cells. ${ }^{*} p<0.05$ versus RANKL-treated alone cells.

\subsection{Fucoidan Prevented Lipopolysaccharide (LPS)-Induced Bone Loss in Mice}

The bone morphometric examination showed that severe bone loss was observed in LPS-treated mice compared to that in control mice (Figure 5A). The images of microcomputer tomography (micro-CT) also confirmed that injection of LPS caused bone loss in mouse femurs reflected by a marked reduction in trabecular bone mass (Figure $5 B$ ). The analysis of various parameters, including bone volume/tissue volume (BV/TV), bone surface/volume ratio (BS/BV), bone mineral density (BMD), average cortical 
thickness for both cortices (Cor.Th), trabecular thickness (Tb.Th.), and trabecular number (Tb.N) used to evaluate the bone loss strongly confirmed that LPS was a potent stimulator of bone loss (Figure 5C). Consistently, treatment with fucoidan $(150 \mathrm{mg} / \mathrm{kg}$ body weight) significantly reduced the extent of bone loss caused by LPS, indicating that fucoidan was able to prevent inflammatory bone loss in vivo.

(A)

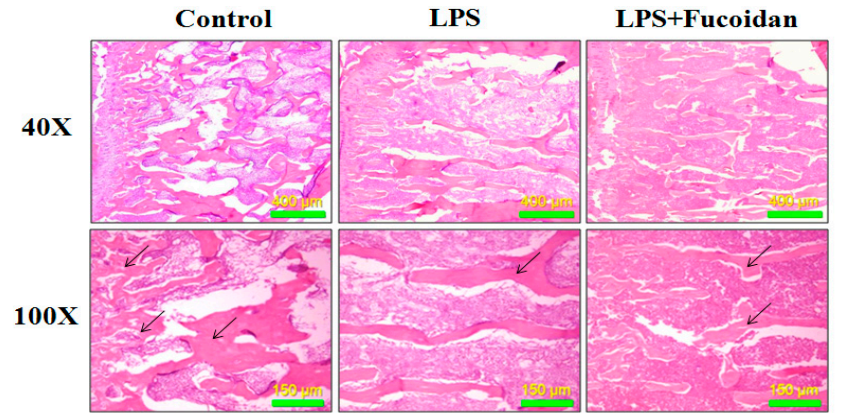

(B)
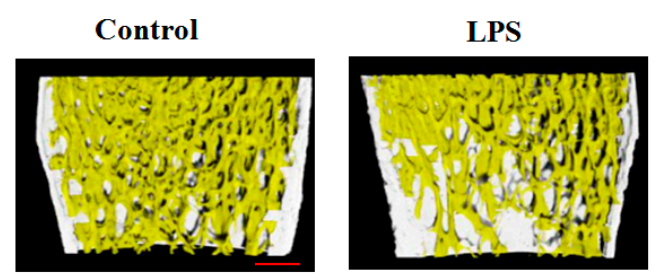

LPS + Fucoidan

(C)
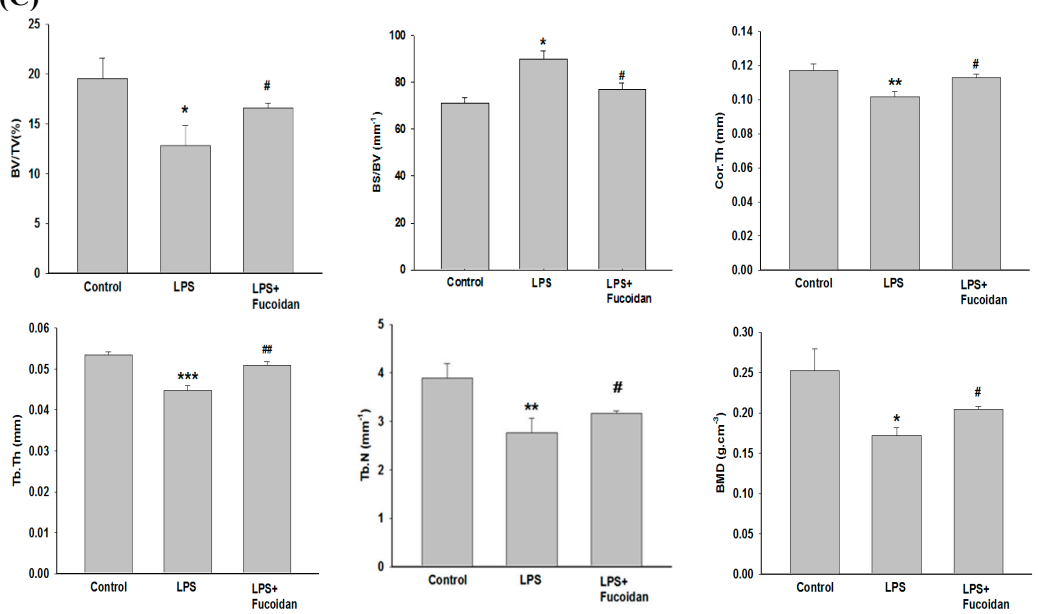

Figure 5. Effects of fucoidan on lipopolysaccharide (LPS)-induced bone loss. Mice were injected intraperitoneally with fucoidan $(150 \mathrm{mg} / \mathrm{kg}$ body weight) or PBS acting as a control group one day before injection with LPS ( $5 \mathrm{mg} / \mathrm{kg}$ body weight). Then, fucoidan or PBS was injected intraperitoneally every other day for eight days. LPS was injected intraperitoneally on day one and four. All mice were killed eight days after the initial LPS injection. The histological changes of femurs were examined by staining with hematoxylin and eosin (H\&E) $(40 \times$ and $100 \times)$ in various groups (scale bar $=150 \mu \mathrm{m})(\mathbf{A})$. The femurs of various groups were scanned with a high-resolution microcomputer tomography (micro-CT). (B) The calculation of the microstructural indices was performed with the micro-CT data (C), including bone volume per tissue volume (BV/TV), bone surface/volume ratio (BS/BV), bone mineral density (BMD), average cortical thickness for both cortices (Cor.Th), trabecular separation (Tb.Sp.), trabecular thickness (Tb.Th.), and trabecular number (Tb.N). Data were expressed as mean \pm SD. ${ }^{*} p<0.05,{ }^{* *} p<0.01$, ${ }^{* * *} p<0.001$ versus control group. ${ }^{\#} p<0.05,{ }^{\# \#} p<0.01$ versus LPS-injected alone group. 


\section{Discussion}

The net effects of osteoblastic bone formation and osteoclastic bone resorption determine the amount of bone mass. Inhibition of excessive osteoclast formation and activity has been considered as a major target for reducing osteolytic bone loss [20]. In this study, fucoidan was proven to be a potent inhibitor on RANKL-induced osteoclastogenesis via regulation of Akt/GSK3 $\beta / P T E N$ and calcium/calcineurin cascades, thereby inhibiting NFATc1 activation. In addition, fucoidan treatment was capable of preventing LPS-induced inflammatory bone loss in vivo.

Our results revealed that increased osteoclast differentiation and bone resorption evidenced by an elevation of TRAP-positive multinucleated osteoclasts, TRAP activity, and Pit formation assay in RANKL-treated macrophages were markedly inhibited by fucoidan. Then, the molecular mechanisms underlying the anti-osteoclastogenic activity of fucoidan were investigated. NFATc1 is a critical transcription factor in promoting RANKL-induced osteoclastogenesis by activating the transcription of genes involved in osteoclast differentiation and activity [21]. RANKL-induced PI3K/Akt activation causing GSK3 $\beta$ phosphorylation and inactivation results in NFATc1 nuclear translocation due to attenuation of NFATc1 phosphorylation [12]. However, GSK3 $\beta$-dependent phosphorylation of PTEN leads to activation of PTEN, which in turn inhibits PI3K/Akt signaling and activates NFATc1 [13]. Therefore, there is a regulatory loop among Akt, GSK3 $\beta$, and PTEN. Fucoidan treatment diminished RANKL-induced phosphorylation of Akt and GSK3 $\beta$ but enhanced PTEN phosphorylation, thereby increasing NFATc1 phosphorylation and suppressing translocation of NFATc1 into the nucleus. However, blocking GSK3 $\beta$ with kenpaullone greatly reversed the effects of fucoidan on NFATc1. Accordingly, the antiosteocalstogenic activity of fucoidan may be associated with NFATc1 inactivation in a GSK3 $\beta$-dependent manner. RANKL also increases intracellular $\mathrm{Ca}^{2+}$ concentration by DNAX-activating protein 12, Fc receptor common chain, and phospholipase $C \gamma$, and subsequently activates calcineurin and NFATc1 [22]. Similarly, the increased $\left[\mathrm{Ca}^{2+}\right]$ i and calcineurin activity observed in RANKL-treated RAW264.7 cells were greatly diminished by fucoidan. Collectively, the inhibitory effect of fucoidan on osteoclast differentiation may be mediated by regulation of the

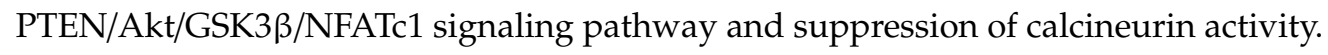

The inflammatory bone resorption occurring in rheumatoid arthritis and periodontitis has been a major clinical problem. Under inflammatory conditions, overproduction of proinflammatory cytokines such as interleukin- 1 and tumor necrosis factor alpha is crucial to trigger osteoclastogenesis and osteolytic bone resorption [23]. Accumulating evidence has indicated that inflammatory cytokine-promoted osteoclastogenesis and bone resorption may be due to enhancement of RANKL production by osteoblast precursors and mature osteoblasts [24,25] and synergy with RANKL to amplify RANKL/RANK-regulated processes [26]. Moreover, the interaction between immune cells and bone cells including osteoblasts and osteoclasts results in an imbalance in bone metabolism by favoring bone resorption [27]. The LPS, a membrane component of Gram-negative bacteria, can enhance the recruitment of various immune cells such as monocytes and macrophages, which further activates the secretion of pro-osteoclastogenic cytokines [28] and preosteoclast fusion and survival [29] that are essential for osteoclast formation and bone resorption. Thus, LPS has been used in most studies to induce inflammatory bone loss in vivo. Fucoidan has been confirmed to exert an anti-inflammatory effect in LPS-stimulated macrophages by suppressing inflammatory cytokine formation and the expression of mitogen-activated protein kinases [30]. Consistent with the results obtained in the in vitro study, treatment with fucoidan significantly mitigated the severity of bone erosion evidenced by an increase in BV/TV, Tb.Th, BMD, and Cor.Th, and a decrease in BS/BV in micro-CT scanning, as well as the examination of bone morphometry in LPS-induced inflammatory bone loss animal model. It has been demonstrated that LPS promotes osteoclast differentiation and the expression of osteoclast-related genes including TRAP, RANK, and MMP-9 in RAW264.7 cells [31]. Thus, suppression of excessive osteoclastogenesis induced by proinflammatory mediators could be a key target to reduce LPS-induced inflammatory bone loss in vivo. Based on the inhibitory effect of fucoidan on osteoclastogenesis in vitro, we expected that there would be similar results in the murine model of inflammatory bone 
loss. Due to the limitation of the bone tissues, the TRAP staining and related target gene expression were not examined in the animal study, which may be evaluated in future studies. Notably, fucoidan has been reported to enhance osteoblast differentiation through activation of ERK, JNK, and bone morphogenetic protein 2 (BMP-2)-Smad 1/5/8 signaling [32]. Moreover, fucoidan increases bone density and bone ash weight in mice due to upregulation of BMP-2, collagen I, and osteonectin, as well as the activity of alkaline phosphatase and osteocalcin $[33,34]$ that are associated with bone mineralization and osteogenesis. Therefore, the ability of fucoidan to enhance osteoblast differentiation and function may be also involved in its protective effect against bone loss by reversing the imbalance in bone metabolism. As fucoidan has several beneficial effects, fucoidan has become a widely used food supplement especially in Asian countries. A recent clinical study reported that patients with metastatic colorectal cancer receiving $4 \mathrm{~g}$ of fucoidan powder twice a day for six months during chemotherapy had a higher disease control rate compared to that of only chemotherapy-treated patients [35]. Thus, oral administration is a potential route of fucoidan administration in patients.

In summary, we demonstrated that fucoidan inhibits osteoclast differentiation and function in vitro and inflammatory bone loss in vivo. The underlying mechanisms accounting for the anti-osteoclastogenic activity of fucoidan may include suppression of calcium/calcineurin cascade and regulation of the Akt/GSK3 $\beta /$ PTEN/NFATc1 signaling pathway (Figure 6). Taken together, fucoidan could be a potential natural regimen for the treatment of osteoclast-related bone diseases.

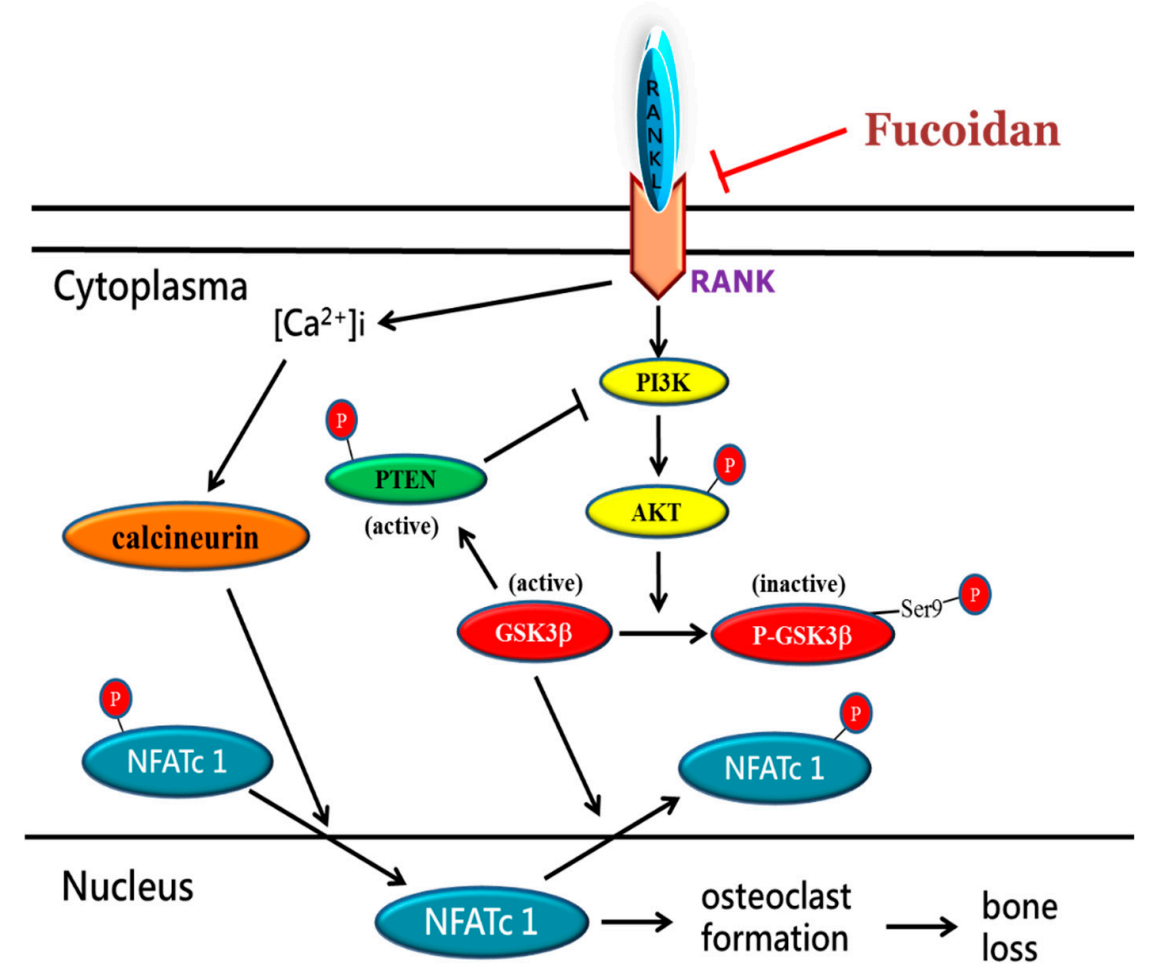

Figure 6. Proposed mechanisms for fucoidan-mediated inhibition of osteoclastogenesis. During RANKL stimulation, fucoidan inhibits Akt phosphorylation leading to GSK3 and PTEN activation, and calcium/calcineurin cascade, which ultimately suppresses nuclear translocation of NFATc1 and osteoclastogenesis-related gene expression, which in turn attenuates osteoclast differentiation.

\section{Materials and Methods}

\subsection{Reagents and Fucoidan Preparation}

The antibodies including anti-phospho-GSK3 $\beta$, anti-GSK3 $\beta$, and anti-phospho-PTEN were purchased from Cell Signaling Technology (Danvers, MA, USA). The anti- $\beta$-actin, anti-NFATc1, and anti-phospho-NFATc1 were purchased from Santa Cruz Biotechnology (Santa Cruz, CA, USA). 
Other chemical reagents used in this study were analytical grade and obtained from Sigma (Saint Louis, MO, USA). The fucoidan was provided by Hi-Q Marine Biotech International Ltd, Taipei, Taiwan) and prepared as described previously [36]. Briefly, dried Sargassum hemiphyllum was incubated with hot water and lyophilized followed by incubation of $95 \%$ ethanol for overnight and then glycolytic enzyme was added. The low molecular weight fucoidan with average molecular weight of $800 \mathrm{Da}$ $(92.1 \%)$ containing fucose $210.9 \pm 3.3 \mu \mathrm{mol} / \mathrm{g}$ and sulfate $38.9 \pm 0.4 \%(w / w)$ was obtained by passing through suitable molecular weight cut-off membranes. The fucoidan dissolved in distilled $\mathrm{H}_{2} \mathrm{O}$ was used for subsequent tests.

\subsection{Cell Culture and Cell Viability Assay}

The mouse RAW 264.7 macrophages (Bioresource Collection and Research Center, Hsinchu, Taiwan) were cultured in $\alpha$-minimal essential medium ( $\alpha$-MEM) (Sigma, St Louis, MO, USA) with $10 \%$ fetal bovine serum. Cell viability was examined by the ability of viable cells to reduce MTT to purple formazan, and the absorbance at $570 \mathrm{~nm}$ was measured by a spectrophotometer (BioTek, Winooski, VT, USA).

\subsection{TRAP Staining and Activity}

RAW 264.7 cells were incubated with RANKL ( $50 \mathrm{ng} / \mathrm{mL}$ ) and fucoidan for 5 days. The cells were fixed with formaldehyde and washed with PBS at room temperature followed by TRAP staining. TRAP-positive multinucleated cells (MNCs $\geq$ three nuclei) were counted under a light microscope (Leica, Vertrieb Deutschland, Germany). The cells for TRAP activity assay were treated as described above. After the cells were fixed with formalin and incubated with ethanol/acetone (1:1) for $1 \mathrm{~min}$, the TRAP solution (50 mM citrate buffer ( $\mathrm{pH} 4.6$ ) containing $10 \mathrm{mM}$ tartrate and $5 \mathrm{mM}$ p-nitrophenylphosphate) was added for $1 \mathrm{~h}$. The reaction was stopped with equal volume of $0.1 \mathrm{~N} \mathrm{NaOH}$. Then, the absorbance was measured at a wavelength of $410 \mathrm{~nm}$ by using a spectrophotometer and the TRAP activity was expressed as a percentage of the activity of RANKL-treated alone cells.

\subsection{Pit Formation Assay}

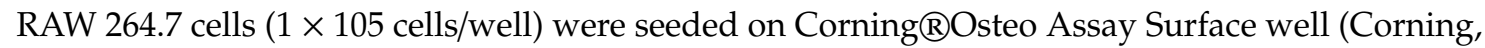
NY, USA) followed by addition of different concentrations of fucoidan and RANKL ( $50 \mathrm{ng} / \mathrm{mL}$ ) for 6 days. Then, cells were removed with $1 \mathrm{~N} \mathrm{NaOH}$ for $20 \mathrm{~min}$. The resorption pit areas were measured by Metamorph imaging analysis (Metamorph Imaging System, Universal Imaging Corp., Downingtown, PA, USA) and the area was expressed as the percentage of the area of RANKL-treated alone group.

\subsection{Western Blotting}

The nuclear and cytosolic protein was extracted by using NE-PER nuclear and cytoplasmic extraction reagents (Thermo Fisher Scientific Inc., Waltham, UT, USA). The protein samples (20-50 $\mu \mathrm{g})$ were separated on $8 \%$ SDS-PAGE gels and transferred to nitrocelluloss membranes. After blocking with $5 \%$ nonfat dry milk in 5\% TBST for $1 \mathrm{~h}$, the membrane was incubated with various primary antibody of target genes $\backslash$ overnight at $4{ }^{\circ} \mathrm{C}$. After washing with TBST three times, the membranes were incubated with a 1:5000 dilution of horseradish peroxidase-conjugated secondary antibody for 1 $\mathrm{h}$ at room temperature. The bands were visualized by using the ECL and chemiluminescence reagent (Milipore, Billerica, MA, USA), and the band intensities were quantified with Image J software (LOCI, University of Wisconsin).

\subsection{Analysis of Intracellular Free $\mathrm{Ca}^{2+}$ Oscillation}

RAW 264.7 cells $\left(1 \times 10^{5}\right)$ pretreated with fucoidan for $2 \mathrm{~h}$ were incubated with $5 \mu \mathrm{M}$ Fluo-4/AM (Thermo Fisher Scientific Inc., Waltham, UT, USA), a calcium indicator fluorescent probe, for $30 \mathrm{~min}$ at $37^{\circ} \mathrm{C}$ in the dark. The fluorescent intensity was measured by using a BD FACSVerse ${ }^{\mathrm{TM}}$ flow cytometry 
at an excitation wavelength of $494 \mathrm{~nm}$ and an emission wavelength of $516 \mathrm{~nm}$ to evaluate the changes of intracellular free $\mathrm{Ca}^{2+}$ oscillation.

\subsection{Calcineurin Activity Assay}

The calcineurin phosphatase activity was measured with a colorimetric calcineurin cellular activity assay kit (Abcam, Cambridge, MA, USA). In brief, cells were lysed using lysis buffer containing protease inhibitors. The protein $(5 \mu \mathrm{g})$ of cell extracts was used to determine the calcineurin activity by measuring the absorbance at $620 \mathrm{~nm}$ and the amount of phosphate released by calcineurin was calculated using a standard curve.

\subsection{LPS-Induced Bone Erosion in Mouse Femurs}

C57BL/6 mice (8 weeks old) were used in this study and had free access to tap water and standard food. There were three groups of five mice each. Mice were injected intraperitoneally with fucoidan ( $150 \mathrm{mg} / \mathrm{kg}$ body weight) or PBS (control group) 1 day before injection of LPS ( $5 \mathrm{mg} / \mathrm{kg}$ body weight, i.p.). Then, fucoidan or PBS was injected every other day for 8 days and LPS was injected on days one and four [37]. At the end of the treatment, the bone tissues of mice were collected for subsequent tests. The animal experiments were approved by an Institutional Animal Care and Use Committee, National Defense Medical Center, Taipei, Taiwan.

\subsection{Bone Morphometric Analyses}

Mouse femur samples were fixed using $4 \%$ paraformaldehyde and the microcomputer tomography (micro-CT) (Bruker Skyscan 1272, Kontich, Belgium) was used to scan samples at $4.6 \mu \mathrm{m}$ resolution. CT scanning was performed at $70 \mathrm{kVp}$ of voltage, $142 \mu \mathrm{A}$ of current, $1100 \mathrm{~ms}$ of exposure time, and with $0.5 \mathrm{~mm}$ aluminum filter. Reconstruction of sections was carried out with GPU-based scanner software (NRecon) (SKYSCAN). For trabecular bone analysis in secondary spongisa, the reconstructed images of distal femur were isolated. Region of interest was defined as trabecular bone area of 1.0-3.0 mm below growth plate (445 slices). In addition, the trabecular bone and cortical bone were automatically isolated using CTAn software (Version 1.15.4.0, Skyscan) provided with the SKYSCAN analysis tool. The alculation of BMD, the microstructural indices of BV/TV, BS/BV, Tb.Th, trabecular number ( $\mathrm{Tb} . \mathrm{N})$, average cortical thickness for both cortices (Cor.Th), and trabecular space (Tb.Sp) were calculated by using CTAn [37]. The density reference was validated by BMD calibration phantoms $(0.25$ and $0.75 \mathrm{~g} / \mathrm{cm}^{3}$ hydroxyapatite). For illustration, CTVox (Version 3.0, Skyscan) (Billerica, MA, USA) was used to provide 3D image. The analysis of cortical bone was carried out by measuring the average cortical thickness for both cortices. Moreover, bone tissues were removed and fixed in $4 \%$ PFA (Sigma-Aldrich) for 1 day at $4{ }^{\circ} \mathrm{C}$ and were then decalcified in $12 \%$ EDTA. The decalcified bones were embedded in paraffin and sectioned for morphometric examination by staining with haematoxylin and eosin (H\&E).

\subsection{Statistical Analysis}

The data were expressed as the mean \pm SD. The statistical analyses between groups were evaluated by one-way ANOVA with a post hoc Bonferroni test. $P$ values less than 0.05 were considered to be a significant difference. All experiments were independently repeated at least five times.

Author Contributions: T.-C.C. conceived and designed the experiments; S.-H.L. performed the experiments and data analysis; Y.-J.H., data analysis and discussion, revision of the manuscript; K.-C.S., data analysis and discussion; S.-H.L. and T.-C.C. wrote the paper. All authors discussed and approved the paper.

Funding: This study was partially supported by a grant of National Science Council of Taiwan, Republic of China (NSC 97-2320-B-016-008-MY3).

Conflicts of Interest: The authors declare no conflict of interest. 


\section{References}

1. Harada, S.; Rodan, G.A. Control of osteoblast function and regulation of bone mass. Nature 2003, 423, 349-355. [CrossRef] [PubMed]

2. Boyle, W.J.; Simonet, W.S.; Lacey, D.L. Osteoclast differentiation and activation. Nature 2003, 423, 337-342. [CrossRef] [PubMed]

3. Takayanagi, H.; Kim, S.; Koga, T.; Nishina, H.; Isshiki, M.; Yoshida, H.; Saiura, A.; Isobe, M.; Yokochi, T.; Inoue, J.; et al. Induction and activation of the transcription factor NFATc1 (NFAT2) integrate RANKL signaling in terminal differentiation of osteoclasts. Dev. Cell 2002, 3, 889-901. [CrossRef]

4. Asagiri, M.; Sato, K.; Usami, T.; Ochi, S.; Nishina, H.; Yoshida, H.; Morita, I.; Wagner, E.F.; Mak, T.W.; Serfling, E.; et al. Autoamplification of NFATc1 expression determines its essential role in bone homeostasis. J. Exp. Med. 2005, 202, 1261-1269. [CrossRef] [PubMed]

5. Aliprantis, A.O.; Ueki, Y.; Sulyanto, R.; Park, A.; Sigrist, K.S.; Sharma, S.M.; Ostrowski, M.C.; Olsen, B.R.; Glimcher, L.H. NFATc1 in mice represses osteoprotegerin during osteoclastogenesis and dissociates systemic osteopenia from inflammation in cherubism. J. Clin. Investig. 2008, 118, 3775-3789. [CrossRef] [PubMed]

6. Boyce, B.F.; Xing, L. Biology of RANK, RANKL, and osteoprotegerin. Arthritis Res. Ther. 2007, 9 (Suppl. 1), S1. [CrossRef] [PubMed]

7. Crabtree, G.R. Generic signals and specific outcomes: Signaling through $\mathrm{Ca}^{2+}$, calcineurin, and NF-AT. Cell 1999, 96, 611-614. [CrossRef]

8. Hirotani, H.; Tuohy, N.A.; Woo, J.T.; Stern, P.H.; Clipstone, N.A. The calcineurin/nuclear factor of activated T cells signaling pathway regulates osteoclastogenesis in RAW264.7 cells. J. Biol. Chem. 2004, 279, 13984-13992. [CrossRef] [PubMed]

9. Doble, B.W.; Woodgett, J.R. GSK-3: Tricks of the trade for a multi-tasking kinase. J. Cell Sci. 2003, 116, 1175-1186. [CrossRef]

10. Beals, C.R.; Sheridan, C.M.; Turck, C.W.; Gardner, P.; Crabtree, G.R. Nuclear export of NF-ATc enhanced by glycogen synthase kinase-3. Science 1997, 275, 1930-1934. [CrossRef]

11. Jang, H.D.; Shin, J.H.; Park, D.R.; Hong, J.H.; Yoon, K.; Ko, R.; Ko, C.Y.; Kim, H.S.; Jeong, D.; Kim, N.; et al. Inactivation of glycogen synthase kinase-3beta is required for osteoclast differentiation. J. Biol. Chem. 2011, 286, 39043-39050. [CrossRef] [PubMed]

12. Moon, J.B.; Kim, J.H.; Kim, K.; Youn, B.U.; Ko, A.; Lee, S.Y.; Kim, N. Akt induces osteoclast differentiation through regulating the GSK3beta/NFATc1 signaling cascade. J. Immunol. 2012, 188, 163-169. [CrossRef] [PubMed]

13. Jang, H.D.; Noh, J.Y.; Shin, J.H.; Lin, J.J.; Lee, S.Y. PTEN regulation by the Akt/GSK-3 $\beta$ axis during RANKL signaling. Bone 2013, 55, 126-131. [CrossRef] [PubMed]

14. Rodan, G.A.; Martin, T.J. Therapeutic approaches to bone diseases. Science 2000, 289, 1508-1514. [CrossRef] [PubMed]

15. Weitzmann, M.N.; Pacifici, R. Estrogen deficiency and bone loss: An inflammatory tale. J. Clin. Investig. 2006, 116, 1186-1194. [CrossRef] [PubMed]

16. Reid, I.R. Pharmacotherapy of osteoporosis in postmenopausal women: Focus on safety. Expert Opin. Drug Saf. 2002, 1, 93-107. [CrossRef] [PubMed]

17. Wang, Y.; Xing, M.; Cao, Q.; Ji, A.; Liang, H.; Song, S. Biological activities of fucoidan and the factors mediating its therapeutic effects: A review of recent studies. Mar. Drugs 2019, 17, 183. [CrossRef]

18. Kim, Y.W.; Baek, S.H.; Lee, S.H.; Kim, T.H.; Kim, S.Y. Fucoidan, a sulfated polysaccharide, inhibits osteoclast differentiation and function by modulating RANKL signaling. Int. J. Mol. Sci. 2014, 15, 18840-18855. [CrossRef]

19. Kubota, T.; Hoshino, M.; Aoki, K.; Ohya, K.; Komano, Y.; Nanki, T.; Miyasaka, N.; Umezawa, K. NF-kappaB inhibitor dehydroxymethylepoxyquinomicin suppresses osteoclastogenesis and expression of NFATc1 in mouse arthritis without affecting expression of RANKL, osteoprotegerin or macrophage colony-stimulating factor. Arthritis Res. Ther. 2007, 9, R97. [CrossRef]

20. Purdue, P.E.; Koulouvaris, P.; Potter, H.G.; Nestor, B.J.; Sculco, T.P. The cellular and molecular biology of periprosthetic osteolysis. Clin. Orthop. Relat. Res. 2007, 454, 251-261. [CrossRef]

21. Kim, J.H.; Kim, N. Regulation of NFATc1 in Osteoclast Differentiation. J. Bone Metab. 2014, 21, $233-241$. [CrossRef] [PubMed] 
22. Asagiri, M.; Takayanagi, H. The molecular understanding of osteoclast differentiation. Bone 2007, 40, 251-264. [CrossRef] [PubMed]

23. Amarasekara, D.S.; Yun, H.; Kim, S.; Lee, N.; Kim, H.; Rho, J. Regulation of osteoclast differentiation by cytokine networks. Immune Netw. 2018, 18, e8. [CrossRef] [PubMed]

24. Wei, S.; Kitaura, H.; Zhou, P.; Ross, F.P.; Teitelbaum, S.L. IL-1 mediates TNF-induced osteoclastogenesis. J. Clin. Investig. 2005, 115, 282-290. [CrossRef] [PubMed]

25. Lam, J.; Abu-Amer, Y.; Nelson, C.A.; Fremont, D.H.; Ross, F.P.; Teitelbaum, S.L. Tumour necrosis factor superfamily cytokines and the pathogenesis of inflammatory osteolysis. Ann. Rheum. Dis. 2002, 61 (Suppl. 2), ii82-ii83. [CrossRef] [PubMed]

26. Fuller, K.; Murphy, C.; Kirstein, B.; Fox, S.W.; Chambers, T.J. TNF alpha potently activates osteoclasts, through a direct action independent of and strongly synergistic with RANKL. Endocrinology 2002, 143, 1108-1118. [CrossRef] [PubMed]

27. Tanaka, Y.; Nakayamada, S.; Okada, Y. Osteoblasts and osteoclasts in bone remodeling and inflammation. Curr. Drug Targets Inflamm. Allergy 2005, 4, 325-328. [CrossRef] [PubMed]

28. Kim, H.A.; Cho, M.L.; Choi, H.Y.; Yoon, C.S.; Jhun, J.Y.; Oh, H.J.; Kim, H.Y. The catabolic pathway mediated by Toll-like receptors in human osteoarthritic chondrocytes. Arthritis Rheum. 2006, 54, 2152-2163. [CrossRef]

29. Suda, K.; Woo, J.T.; Takami, M.; Sexton, P.M.; Nagai, K. Lipopolysaccharide supports survival and fusion of preosteoclasts independent of TNF-alpha, IL-1, and RANKL. J. Cell. Physiol. 2002, 190, 101-108. [CrossRef]

30. Kim, K.J.; Yoon, K.Y.; Lee, B.Y. Low molecular weight fucoidan from the sporophyll of Undaria pinnatifida suppresses inflammation by promoting the inhibition of mitogen-activated protein kinases and oxidative stress in RAW264.7 cells. Fitoterapia 2012, 83, 1628-1635. [CrossRef]

31. Hou, G.Q.; Guo, C.; Song, G.H.; Fang, N.; Fan, W.J.; Chen, X.D.; Yuan, L.; Wang, Z.Q. Lipopolysaccharide (LPS) promotes osteoclast differentiation and activation by enhancing the MAPK pathway and COX-2 expression in RAW264.7 cells. Int. J. Mol. Med. 2013, 32, 503-510. [CrossRef] [PubMed]

32. Kim, B.S.; Kang, H.J.; Park, J.Y.; Lee, J. Fucoidan promotes osteoblast differentiation via JNK- and ERK-dependent BMP2-Smad 1/5/8 signaling in human mesenchymal stem cells. Exp. Mol. Med. 2015, 47, e128. [CrossRef] [PubMed]

33. Hwang, P.A.; Hung, Y.L.; Phan, N.N.; Hieu, B.T.N.; Chang, P.M.; Li, K.L. The in vitro and in vivo effects of the low molecular weight fucoidan on the bone osteogenic differentiation properties. Cytotechnology 2016, 68, 1349-1359. [CrossRef] [PubMed]

34. Cho, Y.S.; Jung, W.K.; Kim, J.A.; Choi, I.W.; Kim, S.K. Beneficial effects of fucoidan on osteoblastic MG-63 cell differentiation. Food Chem. 2009, 116, 990-994. [CrossRef]

35. Tsai, H.L.; Tai, C.J.; Huang, C.W.; Chang, F.R.; Wang, J.Y. Efficacy of low molecular weight fucoidan as a supplemental therapy in metastatic colorectal cancer patients: A double-blind randomized controlled trial. Mar. Drugs 2017, 15, 122. [CrossRef] [PubMed]

36. Hwang, P.A.; Chien, S.Y.; Chan, Y.L.; Lu, M.K.; Wu, C.H.; Kong, Z.L.; Wu, C.J. Inhibition of Lipopolysaccharide (LPS)-induced inflammatory responses by Sargassum hemiphyllum sulfated polysaccharide extract in RAW 264.7 macrophage cells. J. Agric. Food Chem. 2011, 59, 2062-2068. [CrossRef]

37. Zhai, Z.J.; Li, H.W.; Liu, G.W.; Qu, X.H.; Tian, B.; Yan, W.; Lin, Z.; Tang, T.T.; Qin, A.; Dai, K.R. Andrographolide suppresses RANKL-induced osteoclastogenesis in vitro and prevents inflammatory bone loss in vivo. Br. J. Pharmacol. 2014, 171, 663-675. [CrossRef]

(C) 2019 by the authors. Licensee MDPI, Basel, Switzerland. This article is an open access article distributed under the terms and conditions of the Creative Commons Attribution (CC BY) license (http://creativecommons.org/licenses/by/4.0/). 